\title{
Mastication noise reduction method for fully implantable hearing aid using piezo-electric sensor
}

\author{
Sung Dae $\mathrm{Na}^{\mathrm{a}}$, Gihyoun $\mathrm{Lee}^{\mathrm{b}}$, Qun Wei ${ }^{\mathrm{c}}$, Ki Woong Seong ${ }^{\mathrm{d}}$, Jin $\mathrm{Ho} \mathrm{Cho}^{\mathrm{e}}$ and \\ Myoung Nam Kim ${ }^{\mathrm{f}, *}$ \\ ${ }^{a}$ Department of Medical \& Biological Engineering, Graduate School, Kyungpook National University, \\ Daegu, Korea \\ ${ }^{\mathrm{b}}$ Convergence Research Center for Wellness, Daegu Gyeongbuk Institute of Science \& Technology, \\ Daegu, Korea \\ ${ }^{\mathrm{c}}$ Department of Biomedical Engineering, School of Medicine, Keimyung University, Daegu, Korea \\ ${ }^{\mathrm{d}}$ Department of Biomedical Engineering, Kyungpook National University Hospital, Daegu, Korea \\ ${ }^{\mathrm{e}}$ School of Electronics Engineering, College of IT Engineering, Kyungpook National University, \\ Daegu, Korea \\ ${ }^{\mathrm{f}}$ Department of Biomedical Engineering, School of Medicine, Kyungpook National University, Daegu, \\ Korea
}

\begin{abstract}
.
BACKGROUND: Fully implantable hearing devices (FIHDs) can be affected by generated biomechanical noise such as mastication noise.

OBJECTIVE: To reduce the mastication noise using a piezo-electric sensor, the mastication noise is measured with the piezoelectric sensor, and noise reduction is practiced by the energy difference.

METHODS: For the experiment on mastication noise, a skull model was designed using artificial skull model and a piezoelectric sensor that can measure the vibration signals better than other sensors. A $1 \mathrm{kHz}$ pure-tone sound through a standard speaker was applied to the model while the lower jawbone of the model was moved in a masticatory fashion.

RESULTS: The correlation coefficients and signal-to-noise ratio (SNR) before and after application of the proposed method were compared. It was found that the signal-to-noise ratio and correlation coefficients increased by $4.48 \mathrm{~dB}$ and 0.45 , respectively.

CONCLUSION: The mastication noise is measured by piezo-electric sensor as the mastication noise that occurred during vibration. In addition, the noise was reduced by using the proposed method in conjunction with MATLAB. In order to confirm the performance of the proposed method, the correlation coefficients and signal-to-noise ratio before and after signal processing were calculated. In the future, an implantable microphone for real-time processing will be developed.
\end{abstract}

Keywords: Implantable hearing devices, mastication noise, piezo-electric sensor

\footnotetext{
${ }^{*}$ Corresponding author: Myoung Nam Kim, Department of Biomedical Engineering, School of Medicine, Kyungpook National University, Daegu 41944, Korea. E-mail: kimmn@knu.ac.kr.
}

0928-7329/17/\$35.00 (c) 2017 - IOS Press and the authors. All rights reserved

This article is published online with Open Access and distributed under the terms of the Creative Commons Attribution NonCommercial License (CC-BY-NC 4.0). 


\section{Introduction}

Recently, the number of patients with hearing loss has been increasing owing to the increasing average life expectancy of humans and rapid industrialization. Therefore, hearing assistance devices have been developed utilizing advances in medical techniques. In particular, fully implantable hearing devices (FIHDs) are attracting increasing attention, because they overcome acoustic feedback and cosmetic problems [1-7]. The devices are implanted under the skin of the temporal bone [8-10]. The microphone of the devices, however, can be adversely affected when patients are eating meals. Moreover, the acoustic signals collected by the microphone are attenuated through scattering and absorption by the skin and other tissues [11]. The signals can be distorted, although the vibrations caused by patient mastication are relatively small. Jenkins et al. [12] studied the skin vibration of the temporal bone, which is the anatomical vibration generated by mastication. However, they did not consider the anatomical vibration effect for an implanted microphone. Woo et al. [13] studied anatomical mastication with dual-channel microphones and an adaptive filter. However, the adaptive filter is not suitable for hearing devices owing to its excessive computation, and the microphones cannot be affected by the acoustic sounds. In addition, the methods used can cause biocompatibility issues between the implant and the hearing aid. Normally, the temporal bone is carved when FIHDs are implanted in patients. The mastication noises of the vibration characteristic are caused by the bumping together of the upper and lower jawbones. Piezo-electric sensor is suitable for the measurement of the vibrations generated by mastication owing to the piezo-electric sensor's characteristic.

In this paper, reduction methods of mastication noise are proposed that utilizes the piezo-electric sensor. Mastication noise is caused by the vibration of the upper and lower jawbones. The conventional methods of acquiring mastication noise are not suitable for measuring the noise at FIHDs. The noise can be better measured by a piezo-electric sensor than by conventional methods. The proposed method does not require much computation for real-time processing. To confirm the improved efficiency of the proposed method, the results obtained with the proposed method are compared using correlation coefficients and signal-to-noise ratio.

\section{Method}

\subsection{Experimental model and proposed algorithm}

Figure 1 shows the experimental model. To acquire the mastication noise at the FIHDs, the microphone and piezo-electric sensor are used with the skull model.

The implantable microphone was fabricated with an electric condenser microphone (ECM). The piezo-electric sensor constituted of a rectangular element, plastic housing, and a coaxial cable. The sensors were laminated with epoxy or cyanoacrylate due to fix at around the temple according to Fig. 1 . The microphone was fixed with bone cement to reduce outside noise. The piezo-electric sensor was located beside the microphone for the measurement of equal mastication noise. In addition, the microphone and sensor were covered with $6 \mathrm{~mm}$ of silicone owing to imitate the implanted hearing aid on skull model.

The experiment was conducted in a chamber with background noise of approximately $30 \mathrm{~dB}$ SPL. The masticatory movement was generated using the lower jawbone of the skull model. The model was exposed to $1 \mathrm{kHz}$ pure-tone sounds of approximately $85 \mathrm{~dB}$ SPL through standard speakers (FX120, Fostex). An audio-band measurement and analysis device (Fast Ultra Track 8R, M-Audio) was used to measure each output signal of the microphone and piezo-electric sensor. 


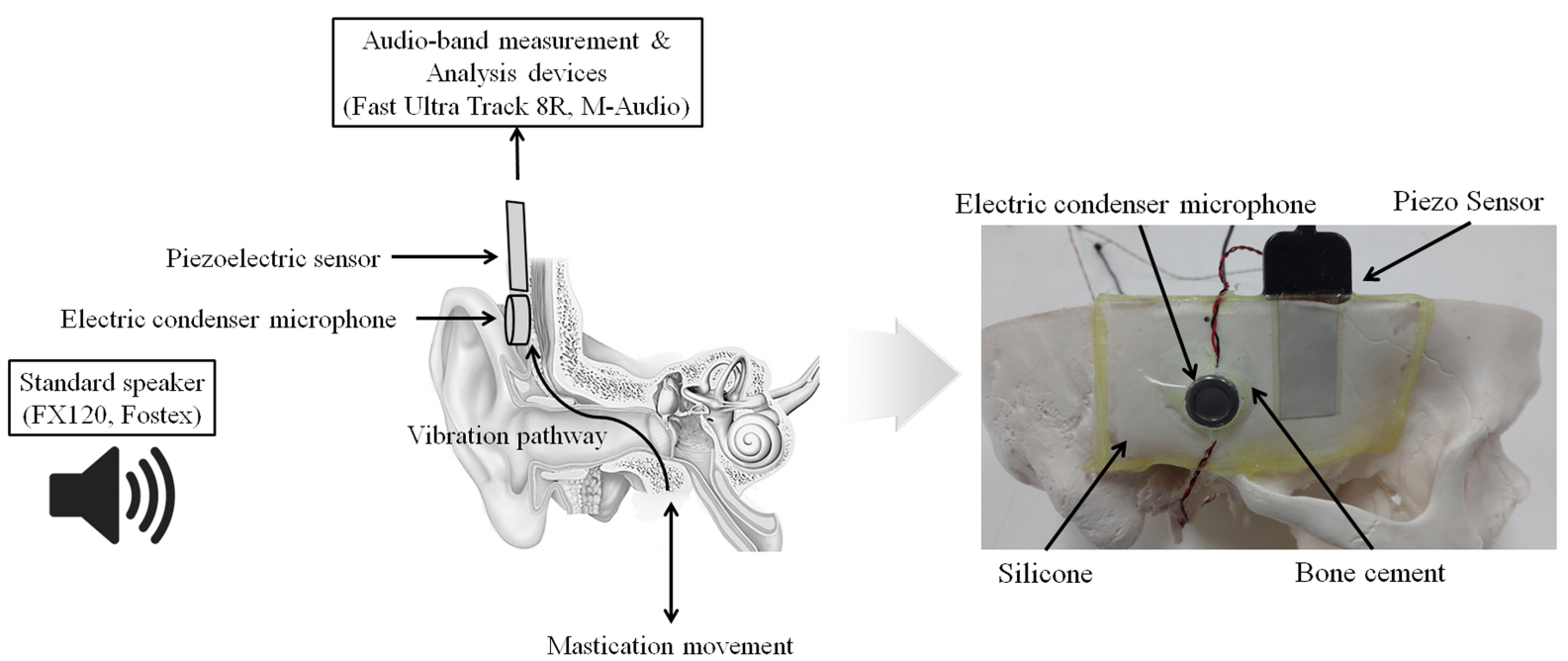

Fig. 1. Experimental model for mastication noise and experimental schematic.

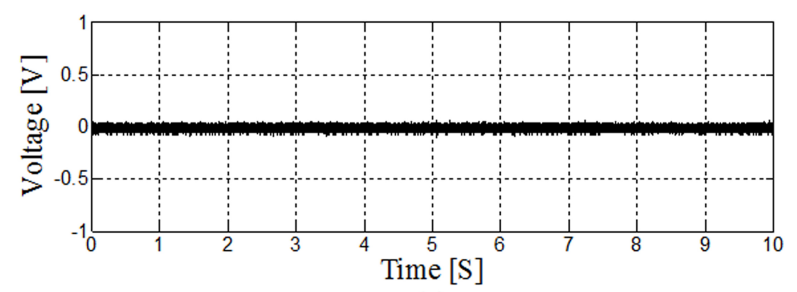

(a)

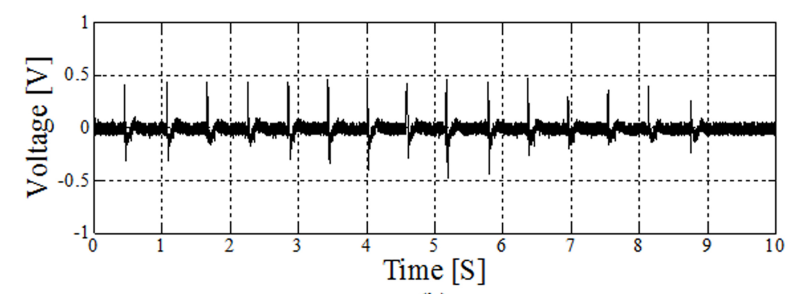

(b)

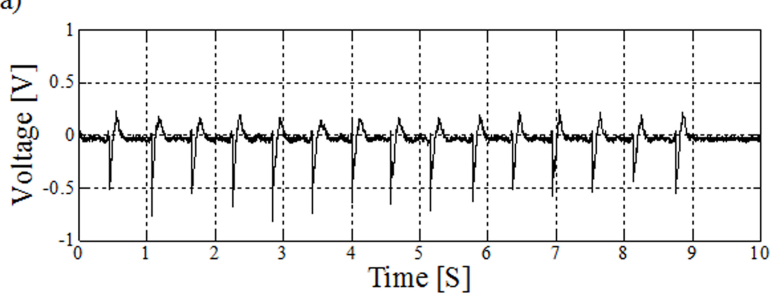

(c)

Fig. 2. Acquired signals with (a) $1 \mathrm{kHz}$ pure-tone at ECM, (b) $1 \mathrm{kHz}$ pure- tone and mastication noise at ECM, and (c) mastication noise at piezo-electric sensor.

The masticatory noises were generated by moving the lower jawbone, and the signals were simultaneously acquired with $1 \mathrm{kHz}$ sounds using a standard speaker. Figure 2 shows the measured signals.

Figure 2(a) presents the appearance of the pure-tone signal. Figure 2(b) shows the simultaneously acquired mastication noise and pure-tone signal. Finally, Fig. 2(c) displays the signals of mastication noise only. The mastication noise mechanism is the vibration of the upper and lower jawbones. The piezoelectric sensor can therefore measure the mastication signal better than the conventional microphone, and hence, it is used in the proposed method.

The ECM under the silicone simultaneously measured both the pure-tone signal and the mastication noise. The noise exhibits greater variation than the pure-tone signals in Fig. 22(c). To reduce the noise, the proposed method employed the following steps. First, the noise ranges were detected by using the 
Table 1

Correlation coefficients and SNR before and after application of proposed method

\begin{tabular}{lcc}
\hline Dataset & Correlation coefficient & SNR \\
\hline Type A (Figs 3[a], 3[c]) & 0.523 & -4.728 \\
Type B (Figs 3[a], 3[d]) & 0.973 & -0.248 \\
\hline
\end{tabular}

standard deviation in the following equation:

$$
N D(t)=\left\{\begin{array}{l}
P S(t)>\sigma, 1 \\
P S(t)<\sigma, 0
\end{array} \text { where, } \sigma=\sqrt{\frac{\sum_{k=1}^{n}\left(x_{k}-m\right)^{2}}{n}}\right.
$$

where $N D(t)$ is the detected noise and non-noise interval gate, $P S(t)$ is the acquired mastication signal of the piezo-electric sensor, and $m$ is the mean of $N D(t)$. The mastication signal exhibits greater variation than the pure-tone signal or other signals. Normally, the standard deviation is useful to extract features from different signals.

The mastication noise was then removed using the proposed method with the acquired pure-tone and mastication signals. Equation (2) shows the proposed method, which uses a nonlinear gain control function:

$$
N F S(t)=\left\{\begin{array}{l}
F S(t) \in N D(t), N F S(t)=\sqrt{\frac{\sum_{k=1}^{n} x_{k}^{2}}{n}-m^{2}} \times \log (F S(t)) \\
\text { else, } \quad F S(t)
\end{array}\right.
$$

where $N F S(t)$ is applied to the proposed method and $F S(t)$ is the signal of the ECM. In this study, the signals measured through the implanted microphone were processed with MATLAB.

\section{Results}

The processed data are shown in Fig. 2. Figure 3(b) shows the noise ranges extracted with the piezoelectric sensor's signal using Eq. (1). In accordance with Eq. (1), the noise components were detected efficiently, as shown in Fig. 1(b). Figure 3(c) shows the simultaneously measured mastication and puretone signals.

Table 1 shows the correlation coefficients before and after the proposed method was applied. Type A is the dataset of Fig 3(a) and (c), and Type B is the dataset of Fig 3(a) and (d). Table 1 shows that the correlation coefficients improved by 0.45 after application of the proposed method. Table 1 shows the SNR before and after application of the proposed method. It is seen that the SNR increases by $4.48 \mathrm{~dB}$ after application of the proposed method. Figure 4 shows that the proposed method's SNR and coefficient are better than those of the conventional method in various SNR environments. However, at $-10 \mathrm{~dB}$ and $-20 \mathrm{~dB}$, lower than before the application of the proposed method due to the high mastication noise and low standard deviation in the noise interval.

\section{Conclusion}

This paper proposed a noise reduction method that employs a piezo-electric sensor to reduce mastication noise. The conventional methods did not consider the biomechanical problems associated with 


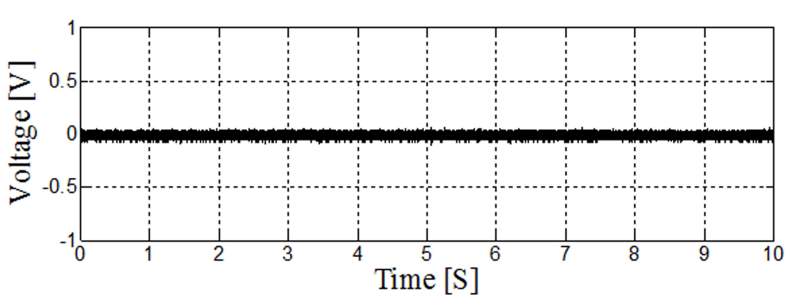

(a)

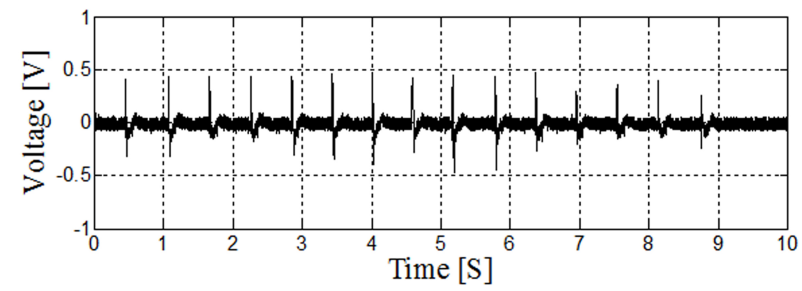

(c)

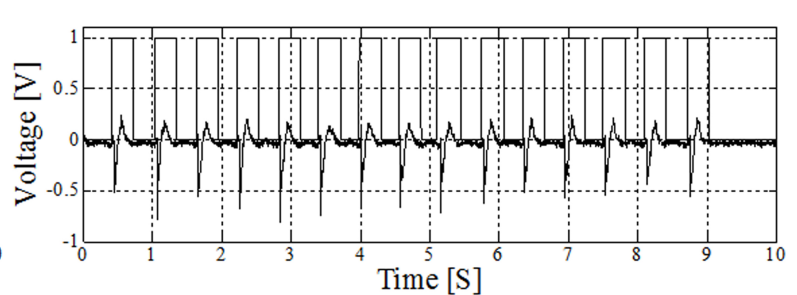

(b)

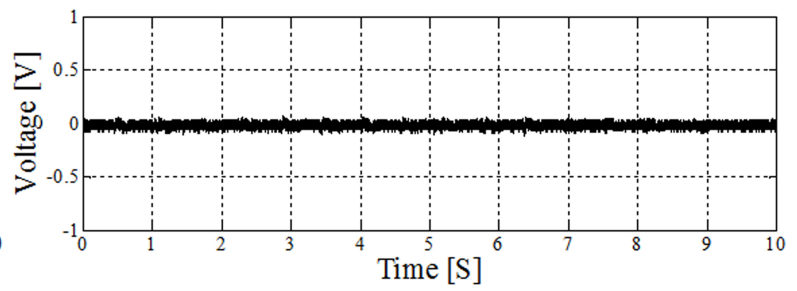

(d)

Fig. 3. Results of experiment and proposed method: (a) $1 \mathrm{kHz}$ pure- tone at ECM, (b) result with Eq. (1), (c) $1 \mathrm{kHz}$ pure-tone and mastication noise at ECM, and (d) result with proposed method.

$\because$ Before Coefficient $\mathbf{x}$ After Coefficient $\square$ SNR

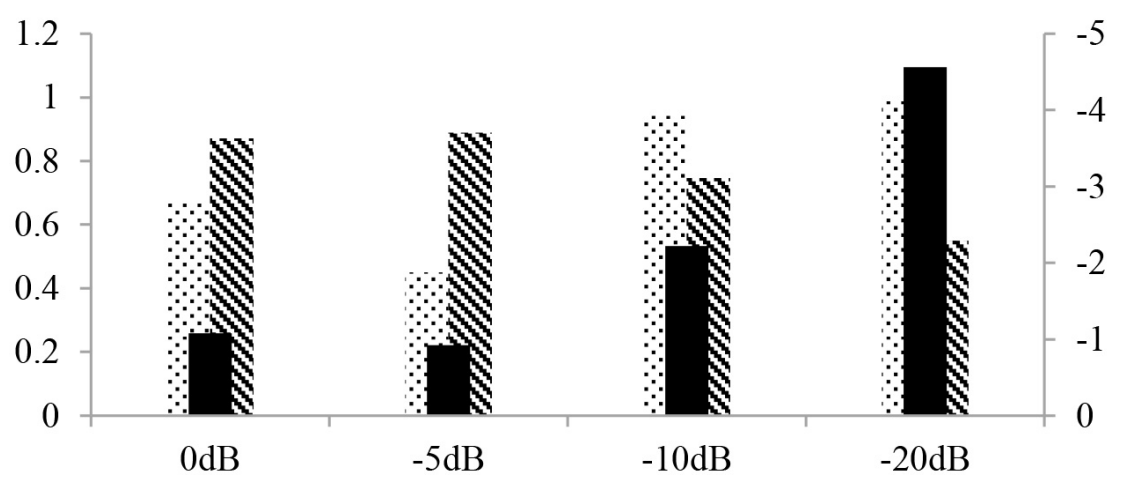

Fig. 4. Results of experiment and proposed method.

mastication movement. Jenkins et al. [12] and Woo et al. [13] studied mastication noise at FIHDs with a microphone. However, because mastication noise is generated as vibrations from the jawbone, the microphone is not suitable because of problems related to biocompatibility. The piezo-electric sensor has the appropriate characteristic to acquire the noise. In this paper, we proposed a method to measure the noise with an implanted microphone and piezo-electric sensor in the model. The noise was reduced by using the proposed method in conjunction with MATLAB. To confirm the performance of the proposed method, the correlation coefficients and SNRs before and after signal processing were calculated. It was found that the SNRs and correlation coefficients increased by $4.48 \mathrm{~dB}$ and 0.45 , respectively. In the future, an implantable microphone for real-time processing will be developed. 


\section{Acknowledgments}

This study was supported by the National Research Foundation of Korea (NRF) grant funded by the Korea government (MSIP) (No. 2016R1A2A1A05005413, 2015R1A2A2A03006113).

\section{Conflict of interest}

None to report.

\section{References}

[1] Spindel JH. Middle ear implantable hearing devices, American Journal of Audiology, 2012; 11: 104-113.

[2] Kochkin S. MarkeTrak VIII: Consumer satisfaction with hearing aids is slowly increasing, Hearing Journal, 2010; 63(1): 19-32.

[3] Jung ES, Seong KW, Lim HG et al., Study on frequency response of implantable microphone and vibrating transducer for the gain compensation of implantable middle ear hearing aid, Journal of Korean Sensors Society, 2010; 19(5): 361-368.

[4] Stenfelt S, Hato N, Goode RL. Fluid volume displacement at the oval and round windows with air and bone conduction stimulation, Journal of Acoustical Society of America, 2004; 115(2): 797-812.

[5] Seong KW, Lim HG, Jung ES et al., Vibration analysis of human middle ear with differential floating mass transducer using electrical model, IEICE Transactions on Information and Systems, 2009; E92-D(10): 2156-2158.

[6] Han JH, Yook S, Nam KW et al., Comparative evaluation of voice activity detectors in single microphone noise reduction algorithms, Biomedical Engineering Letters, 2012; 2: 255-264.

[7] Kim HP, Han JH, Kwon SY et al., Sensitivity enhancement of speech perception in noise by sound training: hearing loss simulation study, Biomedical Engineering Letters, 2011; 1: 137-142.

[8] Han JH, Kim MW et al., Design and fabrication of an implantable microphone for reduction of skin damping effect through FEA simulation, Korea Journal of Biomedical Engineering, 2008; 29: 59-65.

[9] Jung ES, Lim HG, Seong KW et al., Implantable microphone with acoustic tube for fully implantable hearing devices, IEICE Transactions on Information and Systems, 2011; 8(4): 215-219.

[10] Hong EP, Park IY, Seong KW, et al., Application of piezo-electric multi-layered actuator to floating mass transducer for implantable middle ear hearing devices, Journal of Electroceramics, 2009; 23(2): 335-340.

[11] Herman IP. Physics of the human body, Springer, Berlin, Heideler New York, 2007; 555-567.

[12] Jenkins HA, Pergola N, Kasic J. Anatomical vibrations that implantable microphones must oevercom, Otology \& Neurotology, 2007; 28: 579-588.

[13] Woo ST, Jung ES, Lim HG et al., In vivo evaluation of mastication noise reduction for dual channel implantable microphone, Bio-Medical Materials and Engineering, 2014; 24: 239-244. 